\title{
COMPARISON OF FRACTURE BEHAVIOR OF SHARP WITH BLUNT CRACK TIP IN NANOCRYSTALLINE MATERIALS BY MOLECULAR DYNAMICS SIMULATION
}

\author{
MOVAFFAQ KATEB \\ Department of Mining And Metallurgical Engineering, Amirkabir University of Technology, Tehran, Iran \\ Katbab@aut.ac.ir \\ KAMRAN DEHGHANI \\ Department of Mining And Metallurgical Engineering, Amirkabir University of Technology, Tehran, Iran \\ dehghani@aut.ac.ir
}

\begin{abstract}
Molecular Dynamics (MD) simulation was used to figure out the fracture behaviors of nanocrystalline materials (NCM). The simulation was based on more than 13 thousand atoms considered for two systems with sharp and blunt crack tip in NCM. Their atomic level resolution provides novel insights into the fracture behavior of NCM. The results show semi brittle manner for both sharp and blunt tips. Dislocation nucleation and pile up at grain boundary (GB), lead to forming voids at GB. Merging mechanism of voids ahead of crack tip causes crack growth.
\end{abstract}

Keywords: nanocrystalline; molecular dynamics simulation; crack propagation; crack tip; sharp and blunt

\section{Introduction}

Most studies regarding the mechanical properties of NCM exhibit significant differences from coarse-grain materials. ${ }^{1-6}$ For example, it is well known that coarse grain aluminum exhibits ductile fracture behavior due to the extensive slip systems. However, in case of nanocrystalline aluminum, it is reported that twining is the major dominate mechanism. ${ }^{4,5}$

The MD simulation is used in present work because of its strong ability in studying the NCM as preparing a dense nanostructure specimens is not an easy task. ${ }^{5,7}$ Besides, the time and spatial scale involved in both fracture phenomenon and MD technique are compatible, i.e. picosecond and nanometer. In addition, MD has proven to be a significant and powerful approach in modeling the various behaviors of nanostructure materials. ${ }^{8,9}$ For example, many researchers have tried to investigate fracture mechanics using MD simulations. ${ }^{10-13}$ The topics such as instability during dynamic fracture, ${ }^{10,14,15}$ hyperelastic effects in dynamic fracture ${ }^{16}$ intersonic and supersonic cracks, ${ }^{11}$ supersonic crack propagation, ${ }^{17}$ for brittle fracture and crack propagation in FCC materials, ${ }^{18}$ atomic plasticity ${ }^{19}$ crack initiation in FCC materials, ${ }^{20}$ are among the other instances of dynamic 
simulation of ductile fracture. There are also studies on fracture transition mechanism, ${ }^{21}$ brittle and ductile failure in FCC crystals $^{22}$ and fracture behavior of FCC and BCC materials. $^{23}$

So far, the transition from ductile to brittle fracture has been always a concern regarding the $\mathrm{BCC}$ materials. It is well known that sharp and blunt crack resemble the possibility of brittle and ductile fracture, respectively. Generally speaking, the FCC materials, such as aluminum, have been reported to be immune from brittle or cleavage failure. However, it may not be the case for NCM. Therefore, in the present study, using MD simulations, attempts have been made so as to simulate and compare the crack propagation in two modes of sharp and blunt crack in nanostructure FCC materials.

\section{MD Method}

In the MD, the NCMs are supposed as a multi particle system dominated by the classical mechanics. The atoms/particle moves by inter atomic forces exerted from other atoms, and the atom motion is expressed by the Newton's law:

$$
f=m \ddot{r} .
$$

where, $m$ and $\ddot{r}$ are respectively the force vector on the particle, particle mass and acceleration vector. The force vector is determined by gradient of the total energy at a given particle location in space.

$$
f=-\nabla E_{\text {tot }}
$$

where

$$
E_{\text {tot }}=\sum \Phi_{i}
$$

$E_{\text {tot }}$ denotes the total energy of the system which is obtained by adding up the potential energy of each particle. It is assumed that the forces among particles are shortranged, that is only particles within a critical cut-off sphere contribute to the energy of any atom. The cut-off range is chosen to be around the fourth nearest neighbors.

The motion equation (1) is integrated using a velocity verlet scheme, ${ }^{24,25}$ which is proven to be an efficient and reliable method. The velocity verlet algorithm is obtained by a Taylor expansion of the position of particle $i$ at the times of $t_{n+1}$ and $t_{n-1}$. In this scheme, the position vector $\mathrm{r}$ and the velocity vector $\dot{r}$ are defined as follows:

$$
\begin{aligned}
r(t+\Delta t) & =r(t)+\dot{r}(t+\Delta t)+\ddot{r}(t) \Delta t 2 / 2 . \\
\dot{r}(t+\Delta t) & =\dot{r}(t)+[\ddot{r}(t)+\ddot{r}(t+\Delta t)] \Delta t / 2 .
\end{aligned}
$$

The forces at each integrating step are obtained by Equation (2), whereas the acceleration $\ddot{r}$ in Equations (4) and (5) is expressed by $\ddot{r}=f / m$. The interval time $\Delta t$ must be chosen so that the atomic fluctuations can be accurately modeled, and is usually taken in the order of femtosecond for FCC metals such as aluminum, copper and nickel. To simulate the dynamic motion of atoms at the interval time of one nano-second, several thousand integrating steps must be done. 
Depending on which macroscopic parameters are held constant, different ensembles can be defined for the calculation. According to the basic of MD method, the number of particles $N$, the system volume $V$ and the total energy $E$ are held constant. After the initial velocities of the particles are determined using the appropriate MaxwellBoltzmann distribution, the system is simply monitored according to the Newton's law of motion. This corresponds to the micro-canonic $N V E$ ensembles and is more frequently used because it is the most reliable simulation design. However, other ensembles can be desirable for other purposes, for example, the canonical NVT ensemble, where $T$ is absolute temperature, for studying nonequilibrium processes at constant temperature or the isothermal-isobaric NPT ensemble, where $P$ refers to pressure, to investigate the phase transformation. There are several subtleties associated with the NVT and NPT assemblies. ${ }^{24}$ Our simulations were carried out using the $N V E$ ensembles.

For description of inter-atomic forces, a pair potential as a function of a distance between two atoms is the most common one for the $2 \mathrm{D}$ systems. One of the most practical types of potentials is the Lennard-Jones $(\mathrm{LJ})$ used in the present work. ${ }^{24}$

$$
\Phi(r)=4 \varepsilon_{0}\left[(\sigma / r)^{12}-\left(\sigma / r^{6}\right)\right]
$$

where $\varepsilon_{0}$ and $\sigma$ are respectively depth of the minimum of the LJ potential and cut-off distance when the value of the inter-atomic separation and the LJ potential is zero. Besides, we have chosen the LJ potential to capture the generic features of FCC metals. Using a simple LJ potential is also preferred for numerical applications. ${ }^{19}$

The consequence of using LJ potential is that only partial dislocations are nucleated from the cracks, since the stacking fault energy is virtually zero. ${ }^{24}$ Recent studies, however, indicate that partial dislocations play the governing role in the deformation of nano-structure materials (NSM). ${ }^{25,26}$ Therefore, our simulation could be also regarded as a model related to NSM. Besides, all parameters are expressed in reduced units. Lengths are scaled by the LJ parameter $\sigma$ which is assumed unit here, and energies are scaled by the parameter $\varepsilon_{0}=1$. The reduced temperature is $k T / \varepsilon_{0}$ when $k$ is Boltzman constant. The mass is scaled by the atomic mass considering as $m=1$. A cut-off distance equal to 2.5 is assumed for the LJ potential. Reduced time, $t^{*}$ is related to $t$ according to $t^{*}=$ $t\left(\varepsilon_{0} \sigma^{2} / m\right)^{1 / 2}$ and $\Delta t=0.003 t^{*}$.

In $2 \mathrm{D}$ systems, the above potential forms a stable hexagonal lattice, similar to (111) plane of a FCC lattice, which is isotropic under small deformation.

\section{Atomistic Modelling Details}

The dimensions of the system to be simulated in present study were $120 \mathrm{~d}$ and $120 \mathrm{~d}$, where $d$ is inter-atomic spacing, considered as a 2D system of more than 13000 atoms. To perform the MD modeling, the system was assumed as defect free crystals ${ }^{9}$ with amorphous GB that simulated in coarse grain FCC materials. ${ }^{27,28}$ The GB prepared with highest possible density to solve problem of experimental specimen that mentioned above. 
Two notches with the length of $\sim 40 d$ were considered in the middle of system at the left-hand boundary. The notches were parallel to the close packed direction. As we cannot assume any circle or straight line at atomic level, therefore two different crack tips were supposed as shown in Fig. 1 to satisfy blunt and sharp cracks. To study the ductile fracture in nanostructure materials, the tip radius of a blunt crack should be more than 3 atomic distance. ${ }^{26}$ In case of sharp crack, the potentials between two crack faces were removed to ensure that there is no interaction between the atoms of two crack surfaces at up and down.

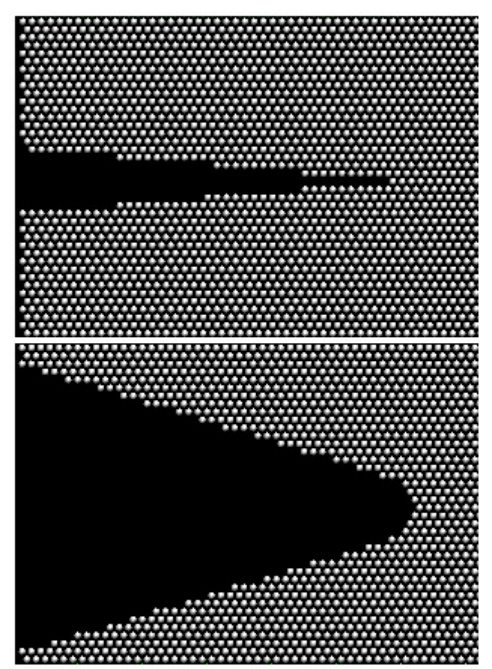

Fig. 1. The morphology of assumed sharp (up) and blunt (bottom) cracks.

The initial reduced temperature in the model was set to 0.01 . The simulation was done similar to the crack opening displacement (COD) test. Referring to Fig. 2, a tensile reduced velocity of 0.7 was employed on both up and down sides of notch to open it. The applied strain rate remains constant during the simulation. 


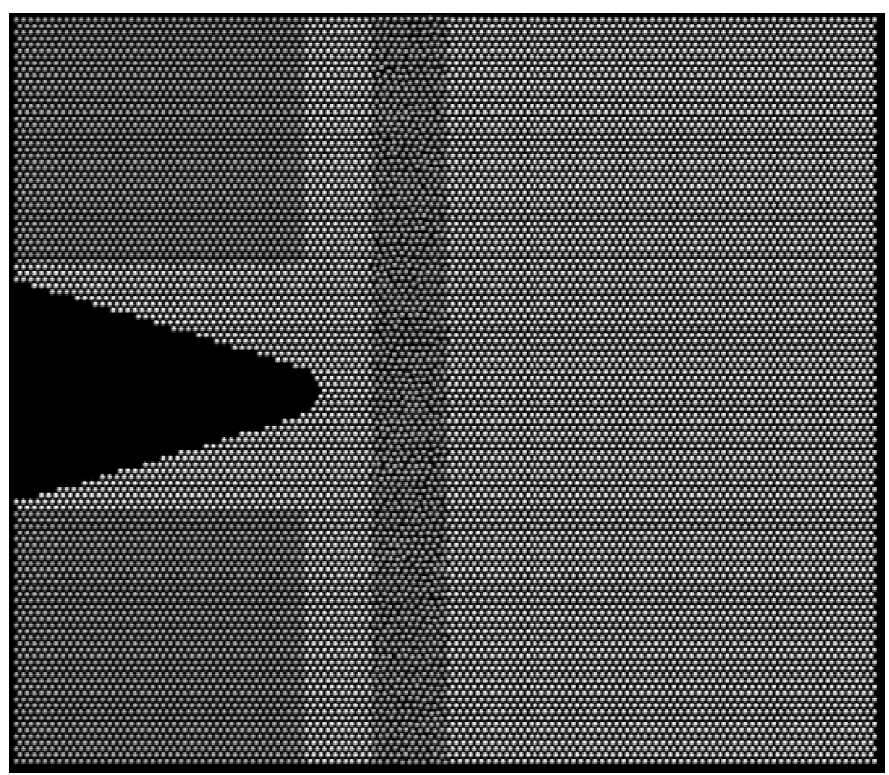

Fig. 2. The $2 \mathrm{D}$ system presenting the notch and grain boundary (dark region ahead of crack). Tensile stress was applied to the darker regions shown above and below the crack.

\section{Results and Discussion}

Fig. 3 shows simulated manner in which sharp and blunt cracks, propagates in NCM for $5,10,15$ and 20 thousand time steps $(\Delta t)$. It also illustrates the changes occurred in sharp and blunt tips as they advance. This was a significant finding that even in case of FCC NCMs, the brittle fracture cannot occur and it is worth mentioning that NCMs is supposed to be immune from brittle fracture.

GBs have considerable fraction in NCMs, as it is obvious from Fig. 3, both sharp and blunt cracks propagation limited by GBs. Unlike coarse grain materials, here crack cannot across the GB and propagate inter granular. The crack will be able to across GB by increasing its velocity, but this is impossible in NCM because, the crack change its path alternatively within GBs and cannot reach to high velocities. It is in agreement with experimental studies but they imply on impurity at $\mathrm{GBs}^{4}$, however this study have no impurity so inter granular fracture is due to amorphous structure of GBs. According to thermodynamics crack select the most unstable path to growth and reduce system energy as much as possible.

To understand the manner of crack in NCM one of the major characteristic is surface roughness/smoothness. Fig. 3 also shows crack surface roughness at atomic level but these surface fluctuations at maximum reach to tenth of nanometer due to inter grain fracture, so considering surface aspect factor, crack propagation in NCMs is not ductile fracture. Also the crack velocity in NCMs, is not in order of brittle fracture. ${ }^{4}$ So the crack propagation in NCM shows semi brittle manner which is in agreement with literatures. ${ }^{4,5}$ 
The major difference between our simulation and previous experimental studies is that, in experimental studies there are many problems which complicate fracture analysis, e.g. inclusions, voids and etc but in present study include no defect except GB. So this simulation most be regarded as defect free study of NCMs.
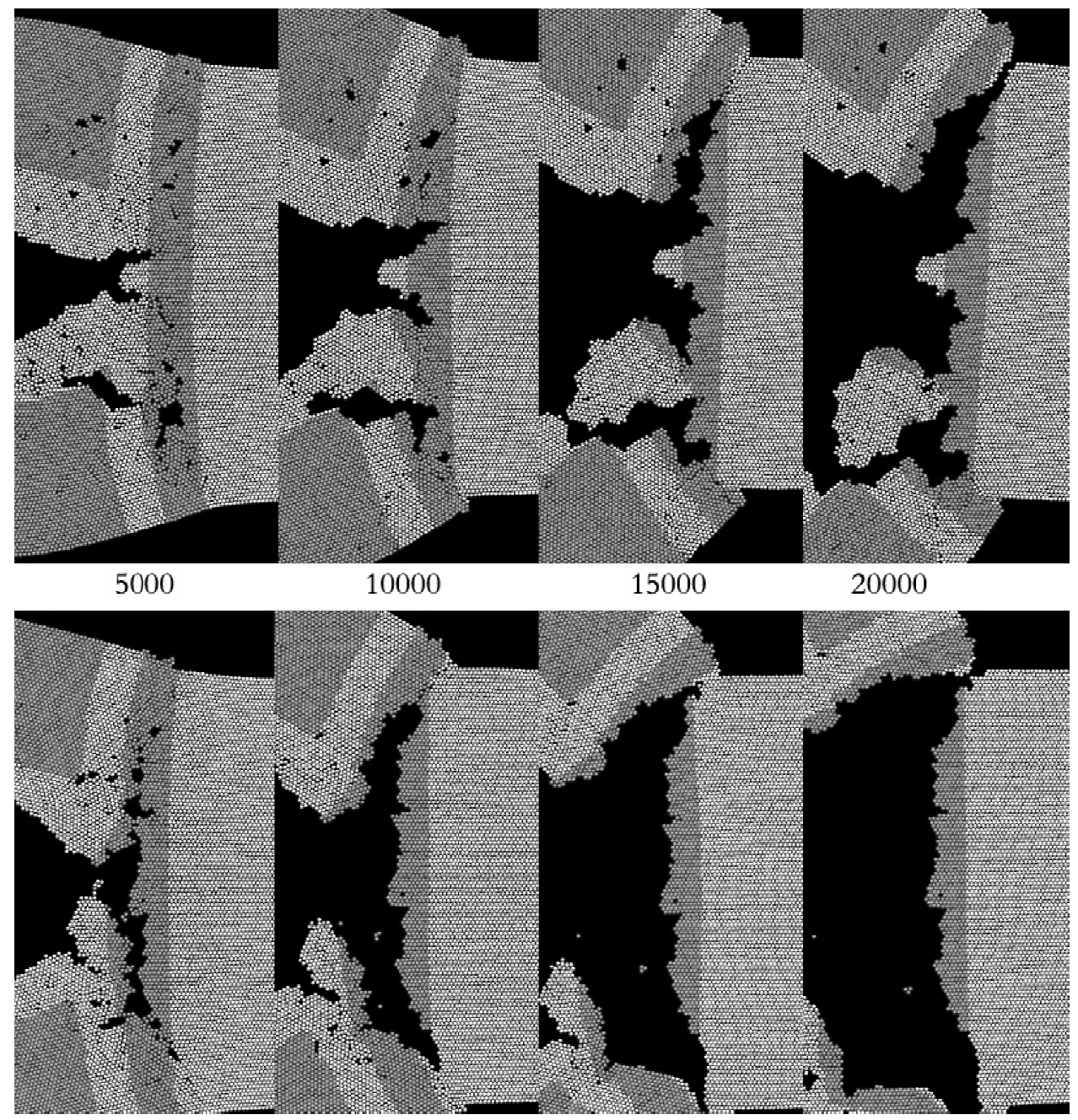

Fig. 3. Crack propagation steps of sharp (upper) and blunt (lower) cracks at 5, 10, 15 and 20 thousand time steps.

There was no way to verify existence of in plain dislocations in 2D simulation yet, but in logical view, because of periodic boundary condition in thickness it can occur. Periodic boundary condition in MD simulation was applied by copying original system around it, so our simulation is not $2 \mathrm{D}$ actually. Fig. 4 shows simple method that verifies existence of dislocation in our 2D simulation. Notice that there are two plans are in front 
and behind of our figures that simulated but not visualized. The bond structure (Fig. 4a) shows displacement and burgers vector, whereas Vacancies and voids were illustrated well in atomic structure (Fig. 4b).

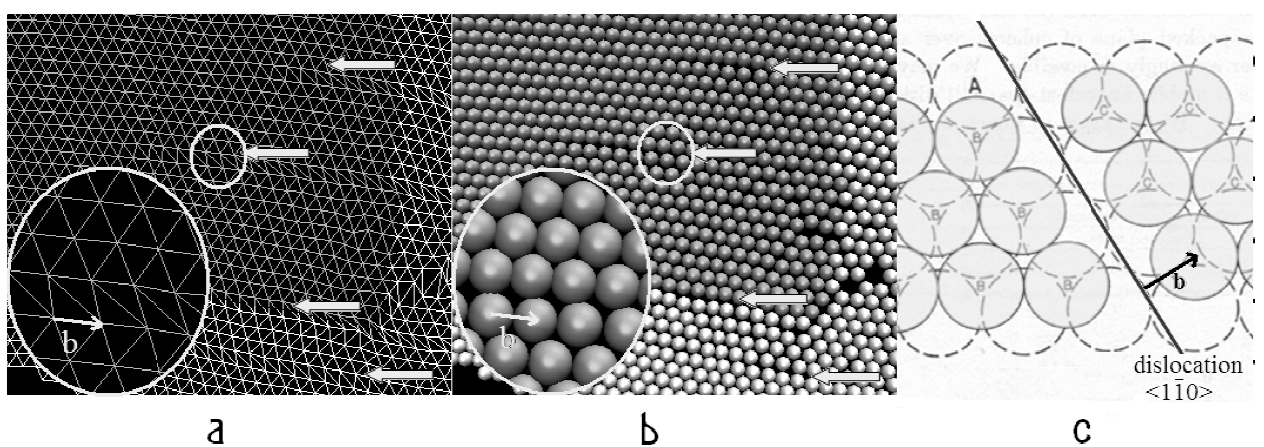

Fig. 4. Illustration of dislocations and burgers vector in a) bond and b) atomic structure at 1900 time steps. White arrows indicate partial dislocations which showed in c) scheme partial dislocation.

In order for plastic deformation to proceed under the imposed stress, the nucleation of dislocations is necessary. Once the pile-up is reached under continuous loading, the emission of dislocations from the crack tip occurs resulting in the relaxation of stress concentration. However, in order to study the emission of dislocation loops and dislocation interactions in more details, a much larger scale of molecular dynamic simulations by use of three dimensional models is necessary.

Around the crack tip, the only operating mechanism is dislocation pile-up leading to stress concentration. In other words, during the initial stages of loading, the dislocations are nucleated. This is followed by their movement around the crack tip resulting in plastic dissipation. Consequently, an increase in plastic work for crack propagation is required that delays the fracture. Upon the continuation of loading, the dislocation density approaches its critical level and pile-up is reached. If it is not relaxed again, then it pronounces the crack propagation and consequently enhances the onset of failure.

In the present work, GB acts as a barrier for dislocation movement. This implies that dislocations are considered free to move until reach to GB and there pile up occurs. Dislocation pile up lid to void formation at GB and voids will guide the crack. However, the dislocations interaction may occur in any place and voids formation will be take place there. Fig. 3 also illustrates some voids which formed in other place except GB.

\section{Conclusions}

In the present study, the MD simulation was used to model the dynamic crack propagation in nanocrystalline FCC materials. Two cases of sharp and blunt crack were investigated in a defect free NCM with inserted grain boundary at amorphous state. Using atomic resolution technique, it is shown that sharp and blunt cracks exhibit similar mechanisms of crack propagation. The dislocation formation and pile up at GB was the 
major mechanism of void formation which guide crack to propagate. A semi-brittle behavior was observed because crack surface shows low roughness seems brittle manner but crack velocity is not in order of brittle fracture.

The results also are acceptable for 3D systems because of periodic boundary condition were applied. But greater model with more atoms and grain boundaries can be more acceptable.

\section{References}

1. T. Mukaia, M. Kawazoeb, K. Higashi, Nanostruct. Mater.10, 755 (1998).

2. M. A. Haque, M. T. A. Saif, Scr. Mater. 47, 863 (2002).

3. M. A. Haque, M. T. A. Saif, Thin Solid Films 484, 364 (2005).

4. M. A. Meyers, A. Mishra, D. J. Benson, Prog. Mater. Sci. 51, 427 (2006).

5. K. S. Kumar, H. Van Swygenhoven, S. Suresh, Acta Mater. 51, 5743 (2003).

6. E. Danaf, S. R. Kalidindi, R. D. Doherty, Int. J. Plast. 17, 1254 (2001).

7. D. Wolf, V. Yamakov, S.R. Phillpot, A. Mukherjee, H. Gleiter, Acta Mater. 53, 1 (2005).

8. M. J. Buehler, H. Gao, Y. Huang, Theor. Appl. Fract. Mech. 41, 21 (2004).

9. I. F. Golovnev, E. I. Golovneva, V. M. Fomin, Comput. Mater. Sci. 37, 336 (2006).

10. M. Marder, Comput. Sci. Eng. 1, 48 (1999).

11. H. Gao, Y. Huang, F. Abraham, J. Mech. Phys. Solids 49, 2113 (2001).

12. M. J. Buehler, H. Gao, Y. Huang, Comput. Mater. Sci. 28, 385 (2003).

13. F. Abraham, et al, Modell. Simul. Mater. Sci. Eng. 6, 639 (1998).

14. F. Abraham, D. Brodbeck, R. Rafey, W. Rudge, Phys. Rev. Lett. 73, 272 (1994).

15. M. Marder, S. Gross, J. Mech. Phys. Solids 43, 1 (1995).

16. M. J. Buehler, F. F. Abraham, H. Gao, Nat. 426, 141 (2003).

17. F. Abraham et al, Proc. Nat. Acad. Sci. U.S.A. 99, 5788 (2002).

18. H. Rafii-Tabar, H.M. Shodja, M. Darabi, A. Dahi, Mech. Mater. 38, 243 (2006).

19. M. J. Buehler, A. Hartmaier, H. Gao, M. Duchaineau, F. F. Abraham, Comput. Methods Appl. Mech. Engrg. 193, 5257 (2004).

20. Y. Shimomura, M. Kiritani, I. Mukouda, Mater. Sci. Engrg. A 350, 238 (2003).

21. K.S. Cheung, S. Yips, Phys. Rev. Lett. 65, 2804 (1990).

22. F. F. Abraham, J. Q. Broughton, Comput. Mater. Sci. 10, 1 (1998).

23. D. Columbus, M. Grujici, Appl. Surf. Sci. 180, 138 (2001).

24. M. Allen, D. Tildesley, Computer Simulation of Liquids, (Oxford University Press, Oxford, 1989).

25. W. C. Swope, H. C. Andersen, P. H. Berens, K. R. Wilson, J. Chem. Phys. 76, 637 (1982).

26. G. E. Dieter, Mechanical Metallurgy, (McGraw-Hill, New York, 1976).

27. H. Van Swygenhoven, A. Caro, D. Farkas, Mater. Sci. Eng. A 309-310, 440 (2001).

28. H. Van Swygenhoven, A. Caro, D. Farkas, Scripta mater. 44, 1513 (2001). 